Bulletin of the Section of Logic

Volume 47/2 (2018), pp. 107-115

http://dx.doi.org/10.18778/0138-0680.47.2.03

Guillermo Badia

\title{
VARIABLE SHARING IN SUBSTRUCTURAL LOGICS: AN ALGEBRAIC CHARACTERIZATION
}

\begin{abstract}
We characterize the non-trivial substructural logics having the variable sharing property as well as its strong version. To this end, we find the algebraic counterparts over varieties of these logical properties.

Keywords: relevant logic, algebraic characterizations of logical properties, variable sharing property, substructural logics.
\end{abstract}

\section{Introduction}

The aim of this note is to fill a gap in Chapter 5 of the, by now, classical reference [4], where the authors deal with a number of logical properties of substructural logics such as the disjunction property, versions of Robinson property, Craig interpolation property, variable separation properties, etc. and their algebraic equivalents on varieties of algebras (following works such as $[6,7,8,11,5]$ and [10] among others). One property in particular is mentioned without providing an algebraic characterization, namely, the variable sharing property (to be defined below). As far as we know, such characterization was not known. Moreover, we provide algebraic counterparts to what is called the strong variable sharing property in [2].

The variable sharing property was first introduced in [1] (pp. 32-33) and it has become since then a folklore necessary (though not sufficient) requirement for any formal system of relevant logic. The philosophical motivation behind it is quite natural: for an implication to be relevant the antecedent better have something in common with the consequent (a recent place where related issues have been studied is [12]). A solid survey where 
this and many other topics in relevant logic are discussed is [3]. A recent place where logics satisfying the requirement have been studied is [9].

These pages grew up from attending [2], where the basic criterion for "relevance" in a given logic was discussed.

\section{Preliminaries}

Our focus will be extensions of the so called "full Lambek calculus" (in symbols, FL). We will be interested in adding absurdity and truth constants $\perp$ and $T$ to our calculi as well. The language of these logics is specified as follows, starting with a collection of propositional variables PROP:

$$
\phi::=p|\perp| \mathrm{T}|1| 0|\psi / \phi| \phi \wedge \psi|\phi \vee \psi| \phi \backslash \psi \mid \phi \cdot \psi,
$$

where $p \in$ PROP. We may write $\phi \cdot \psi$ as $\phi \psi$.

The full Lambek calculus does not have $\perp, T$, so we can give the following Hilbert-style presentation of FL ([4], p. 127):

(id) $\phi \backslash \phi$

(pf) $(\phi \backslash \psi) \backslash((\chi \backslash \phi) \backslash(\chi \backslash \psi))$

(as) $\phi \backslash((\psi / \phi) \backslash \psi)$

(a) $((\psi \backslash \chi) / \phi) \backslash(\psi \backslash(\chi / \phi))$

$(\cdot \vee)((\psi(\psi \backslash \phi)) / \psi) \backslash(\phi / \psi)$

$(\cdot \wedge)((\phi \wedge 1)(\psi \wedge 1)) \backslash(\phi \wedge \psi)$

$(\wedge \backslash)(\phi \wedge \psi) \backslash \phi$

$(\wedge \backslash)(\phi \wedge \psi) \backslash \psi$

$(\backslash \wedge)((\phi \backslash \psi) \wedge(\phi \backslash \chi)) \backslash(\phi \backslash(\psi \wedge \chi))$

$(\backslash \vee) \phi \backslash(\phi \vee \psi)$

$(\backslash \vee) \psi \backslash(\phi \vee \psi)$

$(\vee \backslash)((\phi \backslash \chi) \wedge(\psi \backslash \chi)) \backslash((\phi \vee \psi) \backslash \chi)$

$(\backslash \cdot) \psi \backslash(\phi \backslash \phi \psi)$

$(\cdot \backslash)(\psi \backslash(\phi \backslash \chi)) \backslash(\phi \psi \backslash \chi)$

(1) 1

$(1 \backslash) 1 \backslash(\phi \backslash \phi)$

$(\backslash 1) \phi \backslash(1 \backslash \phi)$

$$
\frac{\phi \phi \backslash \psi}{\psi}(\mathrm{mp}) \quad \frac{\phi}{\phi \wedge 1}(\mathrm{ad}) \quad \frac{\phi}{\psi \backslash \phi \psi}(\mathrm{pn} \backslash) \quad \frac{\phi}{\psi \phi / \psi}(\mathrm{pn} /)
$$


Extending FL with $T, \perp$ can be done by adding axioms $\phi \backslash T$ as well as $\perp \backslash \phi$. Also, when we add the exchange axiom $\phi \psi \backslash \psi \phi$ we obtain a system called $\mathbf{F L}_{e}$.

Notation in this paper will be very much as in [4], except that given an algebra $\mathbf{A}$, we use $\operatorname{dom}(\mathbf{A})$ to denote the domain of $\mathbf{A}$, that is, the universe of the algebra.

An FL-algebra is a structure $\langle A, \wedge, \vee, \cdot, \backslash, /, 1,0\rangle$ such that:

- $\langle A, \cdot, 1\rangle$ is a monoid (i.e., $\cdot$ is associative and 1 is a unit with respect to $\cdot)$

- $\langle A, \wedge, \vee\rangle$ is a lattice (i.e., the operations $\wedge, \vee$ are commutative, mutually absorptive and associative -idempotency is a corollary)

- 0 is some distinguished element of A.

- The residuation law holds: $x y \leqslant z$ iff $y \leqslant x \backslash z$ iff $x \leqslant z / y$ (where, as usual, $x \leqslant y$ iff $x=x \wedge y$ ).

A bounded FL-algebra is obtained from an FL-algebra by adding a top element $T$ and a bottom element $\perp$ (in fact adding a bottom element suffices for $T$ to be defined). We can observe by Lemma 3.6 from [4], that, in fact, every $\mathbf{F L}$-algebra is a subalgebra of a bounded one, hence $\mathbf{F L}$ (which is complete with respect to $\mathbf{F L}$-algebras) is complete with respect to bounded $\mathbf{F L}$-algebras. An $\mathbf{F L} L_{e}$ algebra is an $\mathbf{F L}$-algebra where the multiplication operation · is commutative.

An example of a bounded FL-algebra is the interval $[0,1]$ where $\cdot$ is multiplication on the reals, $\vee$ and $\wedge$ are max and min respectively, $x \backslash y=$ $y / x=\max \{y \in[0,1]: x y \leqslant z\}$, while $1=\top=1$ and $0=\perp=1$.

Given a logic $\mathbf{L}$, the symbol $\mathrm{V}(\mathbf{L})$ denotes the variety corresponding to L. By a substructural logic we will mean a calculus extending FL.

Given a set of propositional variables $X$, by $\operatorname{Fm}(X)$ we denote the set of formulas which can be built from $X$. Finally. given a collection of formulas $\mathbf{F m}(Y)$ based on a list of propositional variables $Y$ and a logic $\mathbf{L}$, by $\mathbf{F m}(Y) / \equiv_{\mathbf{L}}$ we denote the standard Lindenbaum algebra of $\mathbf{L}$.

Definition 1. (VSP) Let $\mathbf{L}$ be some substructural logic. We say that $\mathbf{L}$ has the variable sharing property if given two formulas $\phi$ and $\psi$ where no constants appear, $\vdash_{\mathbf{L}} \phi \backslash \psi$ only if $\operatorname{Var}(\phi) \cap \operatorname{Var}(\psi) \neq \varnothing$.

The next property appears in [2] in a different form where the conjunction involved is the additive $\wedge$ as opposed to the multiplicative $\cdot$. We will split these two properties. 
Definition 2. (SVSP with respect to multiplication) Let $\mathbf{L}$ be some substructural logic. We say that $\mathbf{L}$ has the strong variable sharing property if given formulas $\phi, \psi$ and $\chi$ such that $\vdash_{\mathbf{L}} \phi \psi \backslash \chi$ and $\operatorname{Var}(\psi) \cap \operatorname{Var}(\{\phi, \chi\})=$ $\varnothing$, we have that $\vdash_{\mathbf{L}} \phi \backslash \chi$.

Definition 3. (SVSP^) Let $\mathbf{L}$ be some substructural logic. We say that $\mathbf{L}$ has the strong variable sharing property ${ }^{\wedge}$ if given formulas $\phi, \psi$ and $\chi$ such that $\vdash_{\mathbf{L}}(\phi \wedge \psi) \backslash \chi$ and $\operatorname{Var}(\psi) \cap \operatorname{Var}(\{\phi, \chi\})=\varnothing$, we have that $\vdash_{\mathbf{L}} \phi \backslash \chi$.

The next property is studied on p. 286 of [4].

DeFinition 4. (SDPRP) Let $\mathbf{L}$ be some substructural logic. We say that $\mathbf{L}$ has the strong deductive pseudo-relevance property if given sets of formulas $\Phi, \Psi$ and $\{\chi\}$ such that $\Phi, \Psi \vdash_{\mathbf{L}} \chi$ and $\operatorname{Var}(\Phi) \cap \operatorname{Var}(\Psi \cup\{\chi\})=\varnothing$, we have that if $\Phi \nvdash_{\mathbf{L}} \perp$ then $\Psi \vdash_{\mathbf{L}} \chi$.

Logics with VSP include all systems contained in the relevant logic $\mathbf{R}$, including $\mathbf{F L}, \mathbf{F L}_{e}$ and many extensions (Corollary 5.15 from [4]). A nontrivial example of a system without the variable sharing property is the relevant logic $\mathbf{R M}$.

\section{The results}

In this section we present our little theorems.

THEOREM 1. For any substructural logic $\mathbf{L}$ different from the trivial logic, the following are equivalent:

(i) L has VSP.

(ii) For any cardinals $\kappa, \lambda \leqslant \mu$ there are $\mathbf{A}, \mathbf{B}, \mathbf{C} \in V(\mathbf{L})$ such that $\mathbf{A}, \mathbf{B} \subseteq$ $\mathbf{C}, \mathbf{A}, \mathbf{B}$ and $\mathbf{C}$ are $\kappa$-generated by $A \subseteq \operatorname{dom}(\mathbf{A}), \lambda$-generated by $B \subseteq$ $\operatorname{dom}(\mathbf{B})$ and $\mu$-generated by $C \subseteq \operatorname{dom}(\mathbf{C})$ respectively. Moreover, if $a \in \operatorname{dom}(\mathbf{A}), b \in \operatorname{dom}(\mathbf{B})$ and $a \leqslant_{\mathbf{C}} b$, then there is $\mathbf{D} \in V(\mathbf{L})$ such that $\mathbf{D} \subseteq \mathbf{A}, \mathbf{B}$ and $\mathbf{D}$ is generated by generators in $A \cap B$ appearing in both polynomials $a$ and $b$. 


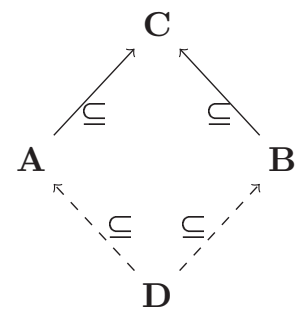

Proof: $(i) \Longrightarrow($ ii $)$ : Consider collections of propositional variables PROP $_{1}$ and $\mathrm{PROP}_{2}$ of cardinalities $\kappa$ and $\lambda$ respectively. Let $\mathrm{PROP}_{3}$ be constructed from PROP $_{1} \cup$ PROP $_{2}$ by possibly adding some new variables to ensure that $\left|\mathrm{PROP}_{3}\right|=\mu$ and put $\mathbf{C}=\mathbf{F m}\left(\mathrm{PROP}_{3}\right) / \equiv_{\mathbf{L}}, \mathbf{A}=\mathbf{F m}\left(\mathrm{PROP}_{1}\right) / \equiv_{\mathbf{L}}$ and $\mathbf{B}=\mathbf{F m}\left(\mathrm{PROP}_{2}\right) / \equiv_{\mathbf{L}}$. By construction of the Lindenbaum algebra we know that $\left\{[p]: p \in \mathrm{PROP}_{3}\right\}$ has cardinality $\mu$ (for otherwise, some $p, q \in$ $\mathrm{PROP}_{3}$ would have to collapse according to $\mathbf{L}$, which would make any two formulas equivalent in $\mathbf{L}$, and hence $\mathbf{L}$ would be the trivial logic), and that this set generates the algebra C. Similarly for $\left\{[p]: p \in \operatorname{PROP}_{1}\right\}$, $\left\{[p]: p \in \mathrm{PROP}_{2}\right\}, \kappa, \lambda, \mathbf{A}$ and $\mathbf{B}$. Also, we clearly have that $\mathbf{A}, \mathbf{B} \subseteq \mathbf{C}$. Now if $a \in \operatorname{dom}(\mathbf{A}), b \in \operatorname{dom}(\mathbf{B})$ and $a \leqslant_{\mathbf{C}} b$ this means that $a=[\phi]$, $b=[\psi]$ for some $\phi \in \mathbf{F m}\left(\mathrm{PROP}_{1}\right), \psi \in \mathbf{F} \mathbf{m}\left(\mathrm{PROP}_{2}\right)$ and in fact $\vdash_{\mathbf{L}} \phi \backslash \psi$. But our assumption that the VSP holds implies that $\operatorname{Var}(\phi) \cap \operatorname{Var}(\psi) \neq \varnothing$, so we can form $\mathbf{F m}(\operatorname{Var}(\phi) \cap \operatorname{Var}(\psi)) / \equiv_{\mathbf{L}}$ as our required $\mathbf{D}$. It is easy to see that $\mathbf{D} \subseteq \mathbf{A}, \mathbf{B}$. Note that $\mathbf{D}$ is generated by $\{[p]: p \in \operatorname{Var}(\phi) \cap \operatorname{Var}(\psi)\}$, which in turn is a subset of $\left\{[p]: p \in \mathrm{PROP}_{1}\right\} \cap\left\{[p]: p \in \mathrm{PROP}_{2}\right\}$

$(i i) \Longrightarrow(i)$ : Suppose that $\vdash_{\mathbf{L}} \phi \backslash \psi$. Recall that this implies that given any homomorphism $h$ from the term algebra under consideration into $\mathbf{E} \in$ $\mathrm{V}(\mathbf{L}), h(\phi) \leqslant_{\mathbf{E}}(\psi)$. In particular, using (ii), pick $\mathbf{A}, \mathbf{B}, \mathbf{C}$ generated by sufficiently large sets such that we can find a homomorphism $h$ from the term algebra into $\mathbf{C}$ such that $h(\phi) \in \mathbf{A}$ and $h(\psi) \in \mathbf{B}$, propositional variables are assigned generators and no different propositional variables get assigned the same image. But then from our assumption that (ii) holds, we must have $\mathbf{D} \in V(\mathbf{L})$ such that $\mathbf{D} \subseteq \mathbf{A}, \mathbf{B}$ and $\mathbf{D}$ is generated by generators in $A \cap B$ appearing in both $h(\phi)$ and $h(\psi)$. But then since $h$ is a homomorphism that assigned different generators to different propositional variables we must have that $\operatorname{Var}(\phi) \cap \operatorname{Var}(\psi) \neq \varnothing$ because $h(\phi)$ and $h(\psi)$ have generators in common. 
TheOREM 2. For any substructural logic $\mathbf{L}$ different from the trivial logic, the following are equivalent:

(i) $\mathbf{L}$ has $S V S P$ with respect to multiplication.

(ii) For any non-degenerate $\mathbf{A}, \mathbf{B} \in V(\mathbf{L})$, there are $\mathbf{C}_{0}, \mathbf{C}_{1}, \mathbf{C}$ such that $\mathbf{C}_{0}, \mathbf{C}_{1} \subseteq \mathbf{C}$ and surjective homomorphisms $h_{0}: \mathbf{C}_{0} \longrightarrow \mathbf{A}$ and $h_{1}: \mathbf{C}_{1} \longrightarrow \mathbf{B}$. Moreover, for any $a, c \in \operatorname{dom}\left(\mathbf{C}_{0}\right)$ and $b \in \operatorname{dom}\left(\mathbf{C}_{1}\right)$ we have that if $a b \leqslant_{\mathbf{C}} c$ then $a \leqslant_{\mathbf{C}} c$. In a picture,

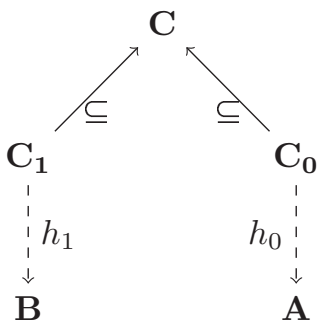

ProOF: $(i) \Longrightarrow(i i)$ : We consider disjoint sets of variables $\mathrm{PROP}_{0}, \mathrm{PROP}_{1}$ big enough such that there will be surjective homomorphisms $f_{0}$ : $\mathbf{F m}\left(\mathrm{PROP}_{0}\right) \longrightarrow \mathbf{A}$ and $f_{1}: \mathbf{F m}\left(\mathrm{PROP}_{1}\right) \longrightarrow \mathbf{B}$ (for definiteness, we name every element of the respective algebra by a propositional variable). Now consider the quotient algebras $\mathbf{F m}\left(\mathrm{PROP}_{0}\right) / \equiv_{\mathbf{L}}, \mathbf{F m}\left(\mathrm{PROP}_{1}\right) / \equiv_{\mathbf{L}} \subseteq$ $\mathbf{F m}\left(\mathrm{PROP}_{0} \cup \mathrm{PROP}_{1}\right) / \equiv_{\mathbf{L}}$. By our assumption that SVSP' holds, obviously $\mathbf{F m}\left(\mathrm{PROP}_{0} \cup \mathrm{PROP}_{1}\right) / \equiv_{\mathbf{L}}$ satisfies that for any $a, c \in \mathbf{F m}\left(\mathrm{PROP}_{0}\right) / \equiv_{\mathbf{L}}$ and $b \in \mathbf{F m}\left(\mathrm{PROP}_{1}\right) / \equiv_{\mathbf{L}}$ we have that if $a b \leqslant c$ in $\mathbf{F m}\left(\mathrm{PROP}_{0} \cup \mathrm{PROP}_{1}\right) / \equiv_{\mathbf{L}}$ then $a \leqslant c$ in $\mathbf{F m}\left(\mathrm{PROP}_{0} \cup \mathrm{PROP}_{1}\right) / \equiv_{\mathbf{L}}$. All that is left is to define surjective homomorphisms $h_{0}: \mathbf{F m}\left(\mathrm{PROP}_{0}\right) / \equiv_{\mathbf{L}} \longrightarrow \mathbf{A}$ and $h_{1}: \mathbf{F m}\left(\mathrm{PROP}_{1}\right) / \equiv_{\mathbf{L}} \longrightarrow \mathbf{B}$. Simply let $h_{i}([\phi])=f_{i}(\phi)(i=1,2)$.

$(i i) \Longrightarrow(i)$ : Suppose that $\vdash_{\mathbf{L}} \phi \psi \backslash \chi, \operatorname{Var}(\psi) \cap \operatorname{Var}(\{\phi, \chi\})=\varnothing$, and that, moreover, $\nvdash_{\mathbf{L}} \phi \backslash \chi$. The latter means that we have some $\mathbf{A} \in V(\mathbf{L})$ such that there is a homomorphism $f_{0}: \mathbf{F m}(\operatorname{Var}(\{\phi, \chi\})) \longrightarrow \mathbf{A}$ such that $f_{0}(\phi) \$ \mathbf{A} f_{0}(\chi)$. We also can find some non-degenerate algebra $\mathbf{B}$ and homomorphism $f_{1}: \mathbf{F m}(\operatorname{Var}(\{\psi\})) \longrightarrow \mathbf{B}$-the value of $h_{1}(\psi)$ will be of little importance. Take $\mathbf{C}_{0}, \mathbf{C}_{1}, \mathbf{C}$ such that $\mathbf{C}_{0}, \mathbf{C}_{1} \subseteq \mathbf{C}$ and surjective homomorphisms $h_{0}: \mathbf{C}_{0} \longrightarrow \mathbf{A}$ and $h_{1}: \mathbf{C}_{1} \longrightarrow \mathbf{B}$. Next we construct $f_{3}: \mathbf{F m}(\operatorname{Var}(\{\psi\}) \cup \operatorname{Var}(\{\phi, \chi\})) \longrightarrow \mathbf{C}$ as follows. First, define:

$$
f_{3}^{\prime}(p)= \begin{cases}\text { some } d \in \operatorname{dom}\left(\mathbf{C}_{0}\right) \text { s.t. } h_{0}(d)=f_{0}(p) & \text { if } p \in \operatorname{Var}(\{\phi, \chi\}) \\ \text { some } d \in \operatorname{dom}\left(\mathbf{C}_{1}\right) \text { s.t. } h_{1}(d)=f_{1}(p) & \text { if } p \in \operatorname{Var}(\{\psi\}) .\end{cases}
$$


Now just extend $f_{3}^{\prime}$ to a homomorphism $f_{3}: \mathbf{F m}(\operatorname{Var}(\{\psi\}) \cup$ $\operatorname{Var}(\{\phi, \chi\})) \longrightarrow$ C. Note that, by assumption, we must have that $f_{3}(\phi) f_{3}(\psi) \leqslant \mathbf{C} f_{3}(\chi)$. But then, since (ii) has been supposed to hold, we have that $f_{3}(\phi) \leqslant \mathbf{C} f_{3}(\chi)$, so, in fact, $f_{3}(\phi) \leqslant \mathbf{C}_{0} f_{3}(\chi)$, but by construction, we would have that also $\left.f_{0} \phi\right) \leqslant \mathbf{A} f_{0}(\chi)$, a contradiction.

Similarly, we can observe that the following holds.

TheOREm 3. For any substructural logic $\mathbf{L}$ different from the trivial logic, the following are equivalent:

(i) $\mathbf{L}$ has $S V S P^{\wedge}$.

(ii) For any non-degenerate $\mathbf{A}, \mathbf{B} \in V(\mathbf{L})$, there are $\mathbf{C}_{0}, \mathbf{C}_{1}, \mathbf{C}$ such that $\mathbf{C}_{0}, \mathbf{C}_{1} \subseteq \mathbf{C}$ and surjective homomorphisms $h_{0}: \mathbf{C}_{0} \longrightarrow \mathbf{A}$ and $h_{1}: \mathbf{C}_{1} \longrightarrow$ B. Moreover, for any $a, c \in \operatorname{dom}\left(\mathbf{C}_{0}\right)$ and $b \in \operatorname{dom}\left(\mathbf{C}_{1}\right)$ we have that if $a \wedge b \leqslant_{\mathrm{C}} c$ then $a \leqslant_{\mathrm{C}} c$.

Proposition 4. For any substructural logic $\mathbf{L}$ extending $\mathbf{F L}_{e}$ with $\perp$, different from the trivial logic, SVSP with respect to multiplication implies $V S P$.

Proof: We modify a proof provided in [2] for a somewhat different context. Assume that $\vdash_{\mathbf{L}} \phi \backslash \psi$ and $\operatorname{Var}(\phi) \cap \operatorname{Var}(\psi)=\varnothing$. Take new propositional variables $p$ and $q$. Now, since obviously $p \backslash p \vdash_{\mathbf{L}} \phi \backslash \psi$, we may conclude, by the local deduction theorem for $\mathbf{F L}$ that there is formula $\theta$ with variables in $\{p\}$ such that $\vdash_{\mathbf{L}} \theta \backslash(\phi \backslash \psi)$ and $p \backslash p \vdash_{L} \theta$. Then $\vdash_{\mathbf{L}} \phi \theta \backslash \psi$ and by SVSP', we have that $\vdash_{\mathbf{L}} \theta \backslash \psi$. Hence, $\psi \backslash \perp_{\mathbf{L}} \theta \backslash \perp$. Therefore, we have that $\psi \backslash \perp, q \backslash q \vdash_{\mathbf{L}} \theta \backslash \perp$. By the local deduction theorem, we have formulas $\delta_{0}, \delta_{1}$ with $\operatorname{Var}\left(\delta_{0}\right) \subseteq \operatorname{Var}(\psi)$ and $\operatorname{Var}\left(\delta_{1}\right) \subseteq \operatorname{Var}(q)$ such that $\psi \backslash \perp \vdash_{\mathbf{L}} \delta_{0}$, $q \backslash q \vdash_{\mathbf{L}} \delta_{1}$ such that $\vdash_{\mathbf{L}} \delta_{0} \backslash\left(\delta_{1} \backslash(\theta \backslash \perp)\right)$. But then also $\vdash_{\mathbf{L}} \delta_{1} \delta_{0} \backslash(\theta \backslash \perp)$, so $\vdash_{\mathbf{L}} \delta_{0} \delta_{1} \backslash(\theta \backslash \perp)$. By an application of SVSP',$\vdash_{\mathbf{L}} \delta_{1} \backslash(\theta \backslash \perp)$. Hence, we can conclude that $\vdash_{\mathrm{L}} \perp$, which is a contradiction.

Now, the argument in [2], shows that, in fact, $\mathrm{SVSP}^{\wedge}$ implies VSP when we can define in our logic $\mathbf{L}$ a negation $\neg$ such that all the following holds for arbitrary $\phi, \psi, \theta$ :

(i) $\vdash_{\mathbf{L}} \phi \backslash \psi$ only if $\vdash_{\mathbf{L}} \neg \psi \backslash \neg \phi$.

(i) For no $\phi$, both $\vdash_{\mathbf{L}} \phi$ and $\vdash_{\mathbf{L}} \neg \phi$.

(ii) Modus ponens for $\backslash$ is an admisible rule.

(i) $\vdash_{\mathbf{L}} \phi \backslash \phi$.

() If $\vdash_{\mathbf{L}} \phi \backslash \psi$ then $\vdash_{\mathbf{L}} \phi \wedge \theta \backslash \psi$. 
Proposition 5. For any substructural logic $\mathbf{L}$ extending $\mathbf{F L}_{e}$ with $\perp$, different from the trivial logic, SVSP with respect to multiplication implies SDPRP.

Proof: Suppose that $\operatorname{Var}(\Gamma) \cap \operatorname{Var}(\Sigma \cup\{\psi\}) \neq \varnothing, \Gamma, \Sigma \vdash_{\mathbf{L}} \psi$ and $\Gamma \nvdash_{\mathbf{L}} \perp$. By the local deduction theorem for FL (Corollary 2.15 from [4]) there are formulas $\gamma$ and $\sigma$ such that $\Gamma \vdash_{\mathbf{L}} \gamma, \Sigma \vdash_{\mathbf{L}} \sigma, \operatorname{Var}(\gamma) \subseteq \operatorname{Var}(\Gamma), \operatorname{Var}(\sigma) \subseteq$ $\operatorname{Var}(\Sigma)$ and $\vdash_{\mathbf{L}} \sigma \backslash(\gamma \backslash \psi)$. So, in fact, $\vdash_{\mathbf{L}} \gamma \sigma \backslash \psi$, and applying SVSP', we must have that $\vdash_{\mathbf{L}} \sigma \backslash \psi$, which in turn means that $\Sigma \vdash_{\mathbf{L}} \psi$ as desired.

As a corollary to this proposition we see that the property described in the characterization of SVSP implies a version of the joint embedding property on subdirectly irreducible bounded $\mathbf{F L}_{e}$ algebras according to Theorem 5.56 from [4].

\section{Conclusion}

We have provided algebraic characterizations for both the variable sharing property and strong variable sharing properties. A line of further research would be to actually apply the characterizations to establish the properties for particular logics, however, it seems like the more traditional method of using matrices is easier in practice (see [9]).

Acknowledgement. I am grateful to the anonymous referee and the editor who provided very helpful corrections to a previous version of this paper. I also acknowledge the support by the Austrian Science Fund (FWF): project I 1923-N25 (New perspectives on residuated posets).

\section{References}

[1] A. R. Anderson and N. D. Belnap, Entailment. The Logic of Relevance and Necessity, I, Princeton University Press, 1975.

[2] A. Avron, The basic relevance criterion, talk given at the Third Workshop in Edmonton, Canada, May 2016.

[3] M. Dunn and G. Restall, Relevance Logic [in:] D. Gabbay and F. Guenthner (eds.), Handbook of Philosophical Logic, Kluwer, 2002, pp. 1-128.

[4] N. Galatos, P. Jipsen, T. Kowalski and H. Ono, Residuated Lattices: An Algebraic Glimpse at Substructural Logics, Elsevier, 2007.

[5] H. Kihara and H. Ono, Algebraic characterizations of variable separation properties, Reports on Mathematical Logic 43 (2008), pp. 43-63. 
[6] L. L. Maksimova, Craig's theorem in superintuitionistic logics and amalgamable varieties of pseudo-Boolean algebras, Algebra i Logika 16 (1977), pp. 643-681.

[7] L. L. Maksimova, On variable separation in modal and superintuitionistic logics, Studia Logica 55 (1995), pp. 99-112.

[8] L. L. Maksimova, Interrelation of algebraic, semantical and logical properties for superintuitionistic and modal logics, Logic, Algebra and Computer Science, Banach Center Publications, Vol. 46, Polish Academy of Science, Warszawa (1999), pp. 159-168.

[9] J. M. Méndez and G. Robles, A General Characterization of the VariableSharing Property by Means of Logical Matrices, Notre Dame Journal of Formal Logic, Vol. 53(2) (2012), pp. 223-244.

[10] D. Souma, An algebraic approach to the disjunction property of substructural logics, Notre Dame Journal of Formal Logic, Vol. 48(4) (2007), pp. 489 495.

[11] A. Wroński, Remarks on Halldén-completeness of modal and intermediate logics, Bulletin of the Section of Logic, Vol. 5/4 (1976), pp. 126-129.

[12] E. Yang, $R$ and Relevance Principle Revisited, Journal of Philosophical Logic 42(5) (2013), pp. 767-782.

Department of Knowledge-Based Mathematical Systems

Johannes Kepler Universität, Austria

e-mail: guillermo.badia_hernandez@jku.at 